\title{
Investigation of ICRF Heating Efficiency in Plug/Barrier Cell on GAMMA 10/PDX with a Full-Wave Code ${ }^{*}$
}

\author{
Yushi KUBOTA, Mafumi HIRATA, Seowon JANG, Makoto ICHIMURA, Ryuya IKEZOE ${ }^{1)}$, \\ Mizuki SAKAMOTO, Shuhei SUMIDA, Atsuto TANAKA, Koki IZUMI, Ryo SEKINE, \\ Hiroki KAYANO, Yousuke NAKASHIMA and Atsushi FUKUYAMA ${ }^{2)}$ \\ Plasma Research Center, University of Tsukuba, Tsukuba 305-8577, Japan \\ ${ }^{1)}$ Research Institute for Applied Mechanics, Kyushu University, Kasuga 816-8580, Japan \\ ${ }^{2)}$ Department of Nuclear Engineering, Kyoto University, Kyoto 615-8540, Japan
}

(Received 30 September 2018 / Accepted 3 December 2018)

\begin{abstract}
In the west end of GAMMA 10/PDX, the end-loss ion flux is utilized for divertor simulation experiments. It is important for controlling parameters of the end-loss ion flux to investigate the efficiency of ion cyclotron range of frequency (ICRF) heating in the west plug/barrier cell, which is next to the west end region. The ICRF heating experiments in the west plug/barrier cell have been performed. In this study, three-dimensional simulations of the ICRF wave propagation were carried out in the plug/barrier cell for the first time. Two types of antennas, a Double Half Turn (WB-DHT) and a Nagoya Type-III (WB-Type-III) antennas, are evaluated. As the results, it is clarified that the WB-Type-III antenna is more effective for the ion heating than the WB-DHT antenna when the frequency is near the ion cyclotron resonance frequency at the midplane of the plug/barrier cell. An additional experiment with the WB-Type-III antenna which is located near the midplane of the plug/barrier cell has been performed. The improvement of the power absorption has been observed. The WB-Type-III antenna located near the midplane is more effective for the ion heating. The experimental results are consistent with the simulation of the wave propagation analysis.
\end{abstract}

(c) 2019 The Japan Society of Plasma Science and Nuclear Fusion Research

Keywords: GAMMA 10/PDX, mirror plasma, ICRF wave, three-dimensional full-wave analysis, ICRF antenna, antenna location

DOI: $10.1585 /$ pfr.14.2401012

\section{Introduction}

GAMMA 10/PDX is a tandem mirror plasma confinement device and it consists of 5 mirror cells and end regions. The end-loss plasma in GAMMA 10/PDX is similar to the scrape off layer (SOL) plasma of largesized Tokamaks. Recently, divertor simulation experiments which utilize end-loss plasmas from the confinement region are implemented in the west end region [1]. The typical end-loss ion parameters at the west end in the normal operations are as follows; the end-loss ion temperature is from 100 to $400 \mathrm{eV}$ and the ion flux is a few in $10^{22} \mathrm{~m}^{-2} \mathrm{~s}^{-1}$ order [2]. In order to advance the divertor plasma study, it is necessary to increase the ion flux to more than 10 times and simulate plasmas in SOL on large-sized tokamaks properly. For increasing the ion flux, experiments with the ion cyclotron range of frequency (ICRF) heating have been carried out by using ICRF antennas installed on GAMMA 10/PDX [2]. In the previous study, it has been observed that the ICRF heating in the west plug/barrier cell, which is next to the west end region, can control the parameters of the end-loss ion effec-

author'se-mail: kubota_yushi@prc.tsukuba.ac.jp

*) This article is based on the presentation at the 12th International Conference on Open Magnetic Systems for Plasma Confinement (OS2018). tively [3].

Toward controlling end-loss ions and extending operational regime for simulating divertor plasmas of large-sized tokamaks, one of the solutions is optimizing ICRF heating in the plug/barrier cell. In order to optimize ICRF heating, it is necessary to investigate the efficiency of the ICRF heating in the plug/barrier cell with the theoretical analysis of the wave propagation and absorption. In this paper, influences of the antenna type and applied frequencies on the wave propagation and absorption in the plug/barrier cell are investigated by using a three-dimensional full-wave code TASK/WF $[4,5]$. In the experiment, an antenna was modified based on the simulation and the improvement of the heating effect has been confirmed.

\section{Experimental Setup}

On GAMMA 10/PDX, plasmas are mainly produced in the central cell, which is located at the center of the device. In anchor cells, which are located on both sides of the central cell, high- $\beta$ plasmas are produced for the magnetohydro-dynamic stabilization of whole plasmas due to the minimum-B configuration. The plug/barrier cells are located at both ends of anchor cells.

Three ICRF oscillator systems called RF1, RF2 and 

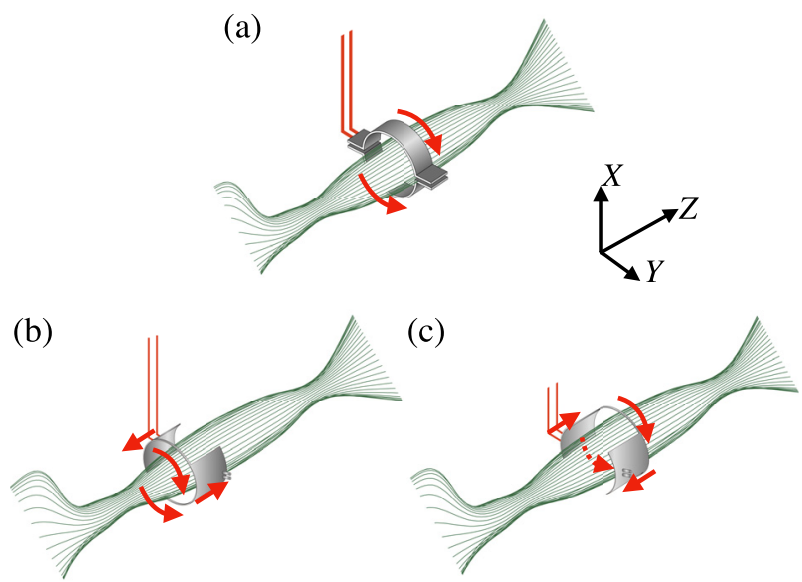

Fig. 1 Schematic drawings of (a) WB-DHT (b) WB-TypeIII and (c) reversed WB-Type-III antennas in the west plug/barrier cell. The red arrows and green lines show the direction of current and magnetic field line, respectively.

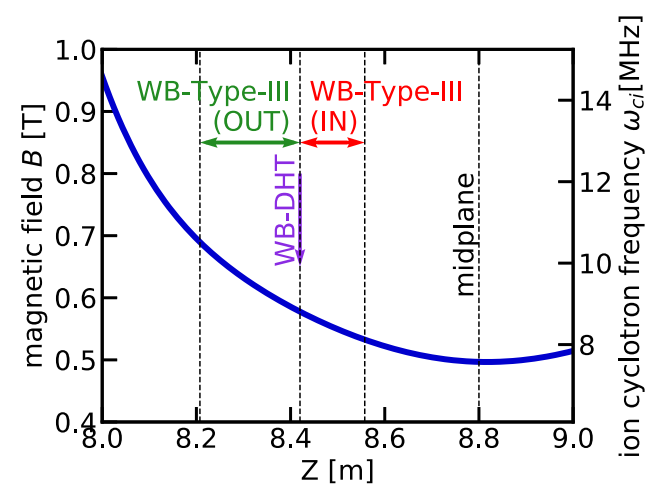

Fig. 2 An axial profile of the magnetic field strength and ion cyclotron resonance frequency near the antenna.

RF3 are used for the experiments. RF1 and RF2 have two sets of power amplifiers and RF3 has a single one. In the normal operation, RF1 is connected to two Nagoya TypeIII (Type-III) antennas located in the central cell and used for the plasma production and heating in the anchor cell. RF2 is connected to two Double Half Turn (DHT) antennas in the central cell and used for the ion heating there.

Two types of antennas, the DHT antenna and the Type-III antenna, are used in the west plug/barrier cell, which called WB-DHT antenna and WB-Type-III antenna, respectively. Schematic drawings of antennas in the west plug/barrier cell are shown in Fig. 1 and the details of the antenna location are shown in Fig. 2 with the distribution of the magnetic field strength and the ion cyclotron resonance frequency, where $Z$ is the distance from the midplane of the central cell in the axial direction. In the case of the WB-DHT antenna, the antenna current flows only in the perpendicular direction to the magnetic field line at $Z=8.42 \mathrm{~m}$ where the resonance frequency is approx- imately $8.8 \mathrm{MHz}$. The normal WB-Type-III antenna is installed in the region of $8.20 \mathrm{~m} \leq Z \leq 8.42 \mathrm{~m}$ where the cyclotron resonance frequency is from $8.8 \mathrm{MHz}$ to $10.5 \mathrm{MHz}$ as shown in Fig. 2. The antenna current flows in the parallel and perpendicular directions as shown in Fig. 1 (b). The reversed WB-Type-III antenna is installed in the region of $8.42 \mathrm{~m} \leq Z \leq 8.57 \mathrm{~m}$ where the cyclotron resonance frequency is from $8.1 \mathrm{MHz}$ to $8.8 \mathrm{MHz}$ as shown in Fig. 2. The reversed WB-Type-III antenna is located nearer the midplane of the plug/barrier cell than the normal WBType-III antenna. The radius of the all antennas is $20 \mathrm{~cm}$. These three types, the WB-DHT, the normal WB-Type-III and the reversed WB-Type-III antennas, are used in this study.

Ion heating efficiency is evaluated in terms of the line density in the plug/barrier cell and the end-loss ion flux with the microwave interferometer in the plug/barrier cell and End Loss Ion Energy Analyzers (ELIEA) [6], respectively. The ELIEA is installed in both end regions and can measure the end-loss ion flux and the energy distribution.

\section{Methods of Simulation}

In order to evaluate the wave propagation and ion heating efficiency, three-dimensional analysis was carried out with TASK/WF code [4,5]. The TASK/WF code solves the following Maxwell's equations with finite elements method.

$$
\nabla \times \nabla \times \vec{E}-\frac{\omega^{2}}{c^{2}} \epsilon \cdot \vec{E}=i \omega \mu_{0} \vec{J}_{\mathrm{ext}}
$$

$\vec{E}$ and $\omega$ are the electric field and the frequency of wave, respectively. $\epsilon$ is the dielectric tensor of the cold plasma including collision effect defined as the following equations.

$$
\begin{aligned}
\epsilon & =\left(\begin{array}{ccc}
S & -i D & 0 \\
i D & S & 0 \\
0 & 0 & P
\end{array}\right), \\
S & =1-\sum_{s} \frac{(\omega+i v)}{\omega} \frac{\omega_{\mathrm{ps}}^{2}}{(\omega+i v)^{2}-\omega_{\mathrm{cs}}^{2}}, \\
D & =\sum_{s} \frac{\omega_{\mathrm{ps}}^{2} \omega_{\mathrm{cs}}}{\omega\left((\omega+i v)^{2}-\omega_{\mathrm{cs}}^{2}\right)}, \\
P & =1-\sum_{s} \frac{\omega_{\mathrm{ps}}^{2}}{\omega(\omega+i v)} .
\end{aligned}
$$

In these equations, $\omega_{\mathrm{ps}}$ and $\omega_{\mathrm{cs}}$ denote the plasma frequency and the cyclotron frequency, respectively. $v$ is the collision frequency. In this code, the cyclotron damping is expressed as the collisional damping. Then, the ion heating efficiency is evaluated with integral of absorption power near the resonance layer. In order to evaluate the ion heating efficiency in the core region, the ion absorption power is integrated in the region of $r_{\mathrm{cc}} \leq 9 \mathrm{~cm}$ and $0.97 \leq B / B_{\mathrm{ci}} \leq 1.03$, where $r_{\mathrm{cc}}$ and $B_{\mathrm{ci}}$ denote the radius converged at the midplane of the central cell and the mag- 


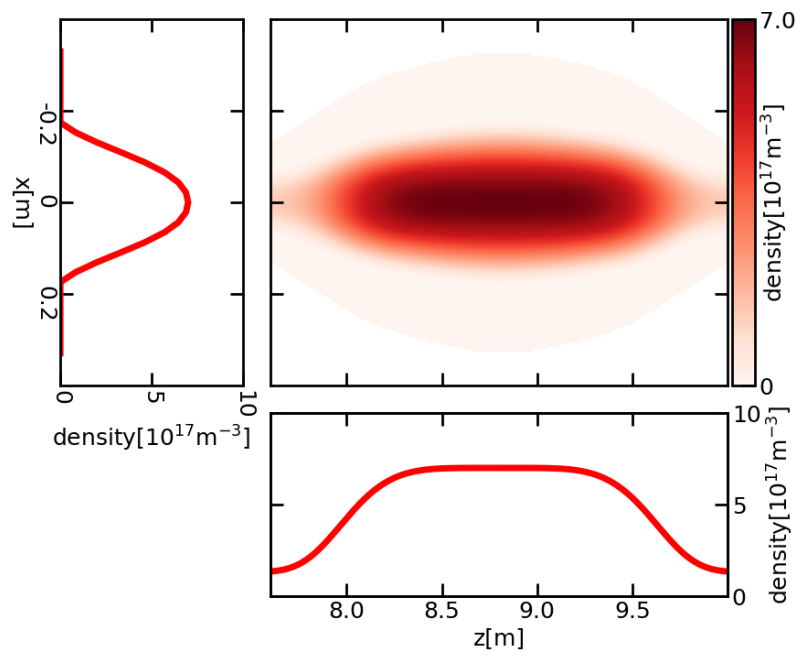

Fig. 3 The density profile in the west plug/barrier cell using TASK/WF code. The density at the midplane is estimated based on the measurement using the interferometer.

netic field strength of the ion cyclotron resonance, respectively. $\vec{J}_{\text {ext }}$ is the external antenna current and the antenna current paths which are input to $\vec{J}_{\text {ext }}$ are shown schematically by the red arrows in Fig. 1 .

The collision frequency affect to the broadness of the simulated resonance layer. If the collision frequency is not adequately high and the simulated resonance layer is not sufficiently broader than the element sizes in axial direction, the absorption power are simulated to a different value by changing the position of the elements and nodes. The ratio of the collision frequency to the wave frequency is set to $4 \times 10^{-3}$ except for the boundaries and confirmed that absorption power is independent of the location of the elements and nodes under the value of collision frequency. The collision frequency is set to be sufficiently high near the axial boundaries in order to prevent the reflection of the waves at the boundaries. In this study, we focused on the wave propagation and absorption only in the plug/barrier cell and set a calculation region between mirror throats of the plug/barrier cell.

Figure 3 shows the density profile using for the simulation with TASK/WF code. The density at the midplane of the plug/barrier cell is estimated to be $7.0 \times 10^{17} \mathrm{~m}^{-3}$ based on the measured line density with the microwave interferometer in the west plug/barrier cell.

\section{Results of Simulation}

In order to compare ion heating efficiencies of the three antennas, frequency dependencies of the wave absorption power are calculated. The calculated frequency dependencies are shown in Fig. 4.

The WB-DHT antenna has a peak of the absorption power around $9.0 \mathrm{MHz}$, at which the resonance layer exists near the WB-DHT antenna. It is considered that ion heat-

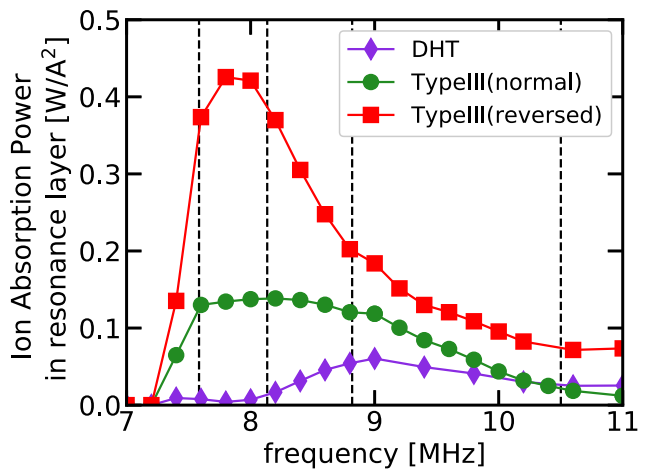

Fig. 4 Frequency dependences of calculated ion absorption powers in the resonance layer in the west plug/barrier cell.

ing efficiency increases near the ion cyclotron frequency in front of the antenna in the case of the WB-DHT antenna. In contrast, the absorption power in the case of the WBType-III antennas has a peak of the absorption power in the frequency range between 7.6 and $9.0 \mathrm{MHz}$, which indicates the absorption power becomes large when the resonance layer is located near the midplane. The existence of the resonance layer near the midplane is more effective for the ion heating than that near the antenna. The gradient of the magnetic field strength near the midplane becomes smaller than that near the antenna location. These results are consistent qualitatively with the results of the experiment with the WB-DHT and the normal WB-Type-III antennas reported in Ref. [3].

As shown in Fig. 4, the absorption power of the reversed WB-Type-III antenna becomes higher than that of the normal WB-Type-III antenna, especially in the case that the resonance layer is located near the midplane. It is remarkable that the ion absorption power of the reversed WB-Type-III antenna is also higher than that of the normal WB-Type-III antenna even in the case of more than 9.0 MHz. One of considerable reasons is that the distance between the antenna and plasma surface becomes small near the midplane when the radius of the antenna is fixed on $20 \mathrm{~cm}$ in the calculation.

\section{Results of Experiments}

From the results of the TASK/WF calculation, we have modified the normal WB-Type-III antenna to the reversed WB-Type-III antenna in the west plug/barrier cell as shown in Fig. 1 (c). The ICRF heating experiments with the reversed WB-Type-III antenna have been carried out under the similar experimental conditions with the normal WB-Type-III antenna. The frequency of RF3 connected to the antennas in west plug/barrier cell is set to $7.7 \mathrm{MHz}$ and the resonance layer is located near the midplane.

Figure 5 shows the time evolutions of the input power of RF3, the line density near the midplane of the west 


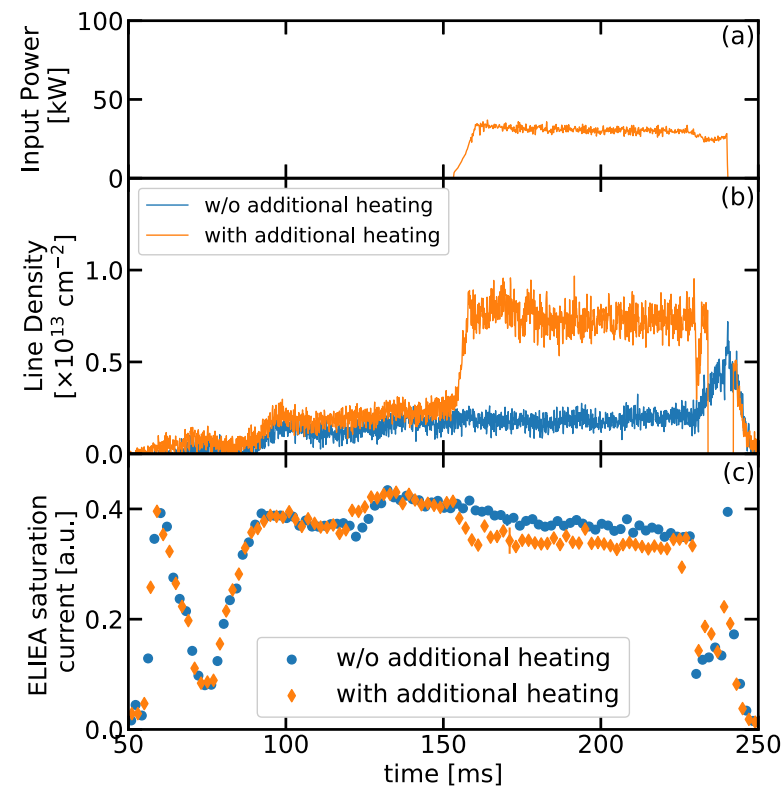

Fig. 5 The time evolutions of (a) input power (b) line density in the west plug/barrier cell (c) ELIEA saturation current in the typical discharges.

plug/barrier cell and the ELIEA saturation current, which is proportional to the end-loss ion flux, in the typical discharges with and without the additional heating using the reversed WB-Type-III antenna. During the additional heating, the line density increased clearly as shown in Fig. 5 (b) while the ELIEA saturation current decreased slightly as shown in Fig. 5 (c).

The RF3 power dependencies of the line density in the west plug/barrier cell and the ratio of the end-loss ion flux measured by ELIEA at the west end during to before applying the additional heating with the reversed WBType-III and the normal WB-Type-III antennas are shown in Fig. 6. The trends of the increase of the line density and the decrease of the end-loss ion flux with the increase of the RF3 power are indicated. The pitch angle scattering of the ions from the loss-cone region of the velocity space in the plug/barrier cell due to the ion cyclotron resonance heating is considered to be one of the possible mechanisms. The increase rate of the line density and the reduction rate of the ion flux with the reversed WB-Type-III antenna tends to be higher than those with the normal WB-Type-III antenna. These results indicate that ion heating efficiency is improved by reversing the installation direction of the WBType-III antenna. It is confirmed that the simulation results with the TASK/WF code shown in Fig. 4 are qualitatively consistent with the experimental results.

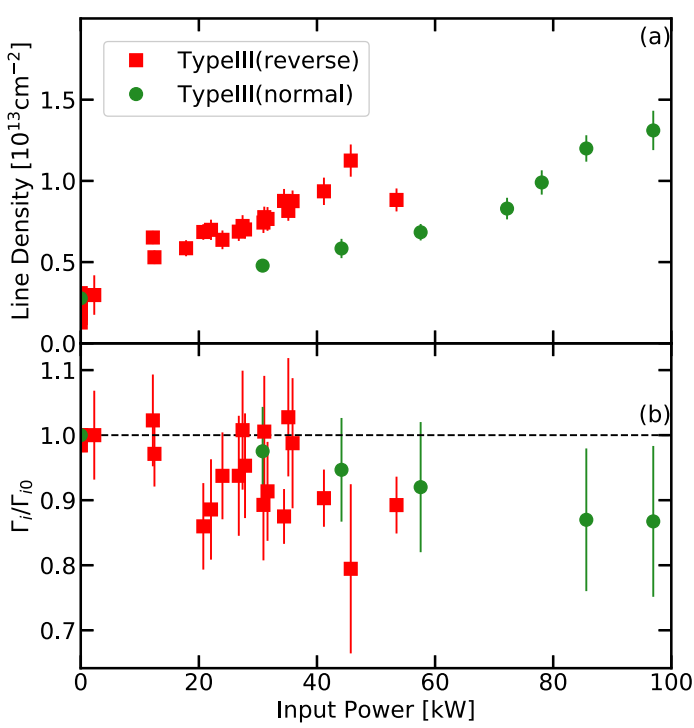

Fig. 6 Power dependencies of (a) line density in plug/barrier cell (b) end-loss ion flux.

\section{Summary}

In order to investigate the ion heating efficiency in the plug/barrier cell on GAMMA 10/PDX, wave excitation, propagation and absorption analysis with TASK/WF code and experiments are carried out. From the simulation results, it is clarified that the reversed WB-Type-III antenna is most effective for ion heating among the WBDHT antenna, the normal and reversed WB-Type-III antennas. The ICRF heating experiments using these antennas are performed. It is confirmed in the experiment that the reversed WB-Type-III antenna improves the heating efficiency, which is consistent with the results of the simulation.

\section{Acknowledgements}

The authors acknowledge the member of plasma research center in university of Tsukuba for their collaboration. This work was partly supported by the bidirectional collaborative research program of the National Institute for Fusion Science, Japan (NIFS14KUGM086 and NIFS17KUGM132).

[1] Y. Nakashima et al., Nucl. Fusion 57, 116033 (2017).

[2] R. Ikezoe et al., Fusion Sci. Technol. 68, 63 (2015).

[3] S. Jang et al., AIP Conf. Proc. 1771, 030011 (2016).

[4] A. Fukuyama et al., Proc. of 1996 Int. Conf. on Plasma Phys. 2, 1342 (1997).

[5] T. Yokoyama et al., Fusion Sci. Technol. 68, 185 (2015).

[6] K. Ichimura et al., Plasma Fusion Res. 7, 2405147 (2012). 